EDITORIAL

\title{
Effects of the COVID-19 pandemic on gender representation among corresponding authors of Neuropsychopharmacology (NPP) manuscripts: submissions during January-June, 2020
}

\author{
Neuropsychopharmacology (2021) 46:269-270; https://doi.org/10.1038/s41386-020-00869-4
}

Neuropsychopharmacology (NPP) is committed to promoting diversity, inclusion, and opportunity in all aspects of its function [1]. As part of our efforts to enhance mindfulness of these goals, we engage in special projects involving data-driven self-evaluation and analyses. In 2018 and 2019, we released reports on gender balance in NPP function $[2,3]$. These reports documented the representation of individuals identified as women or men serving in roles as senior editors, editorial board members, reviewers, or authors at NPP. In addition, the second report examined if procedural changes implemented following the first report had altered trends in these metrics. We found that, over the period of January-June in each of these years, women represented corresponding authors on 38.1 and $39.2 \%$ (respectively) of the manuscripts submitted to NPP. These numbers closely align with the base rate of women in the field of "biological psychiatry," which is currently estimated at $40 \%$ (Biaswatchneuro.com). The field of biological psychiatry was selected for our base rate estimate because it includes a blend of psychiatry and neuroscience, overlapping with the mission and membership of the American College of Neuropsychopharmacology (ACNP), the professional society represented by NPP.

The COVID-19 pandemic has profoundly disrupted research and necessitated large-scale shifts to remote telework. Accompanying these shifts, there have been reports of decreased rates of manuscript submissions, grant submissions, and/or reduced productivity from women [4]. While the causes of this trend are beyond the scope of our analyses-increased burdens related to childcare, household, and personal responsibilities have been proposed as explanations - these reports inspired us to examine whether the gender demographics of authors submitting to NPP had changed during the COVID-19 pandemic.

To determine the impact of COVID-19 on NPP submission demographics, we characterized gender representation among corresponding authors submitting to NPP from January 1, 2020 through June 30,2020, approximating the world-wide onset of the COVID-19 pandemic. We compared these data with those from the same period in 2018 and 2019, which we had collected as part of our previous reports [2,3]. As before, we performed detailed on-line searches for the use of gender-specific pronouns on websites and/or photographs to match an individual's indicated (first) name to their gender.

From January-June 2020, 749 manuscripts were submitted to NPP, compared to 583 submissions during the same time period in 2018 and 608 in 2019 (Fig. 1). Among the 2020 submissions, $38.5 \%$ of corresponding authors of NPP submissions were identified as women, falling almost exactly between the proportions reported for the same period in 2018 and 2019. Thus while NPP submission rates were increased by $\sim 26 \%$ during the period of time overlapping with the early stages of the COVID-19 pandemic and the shut-down of research programs around the

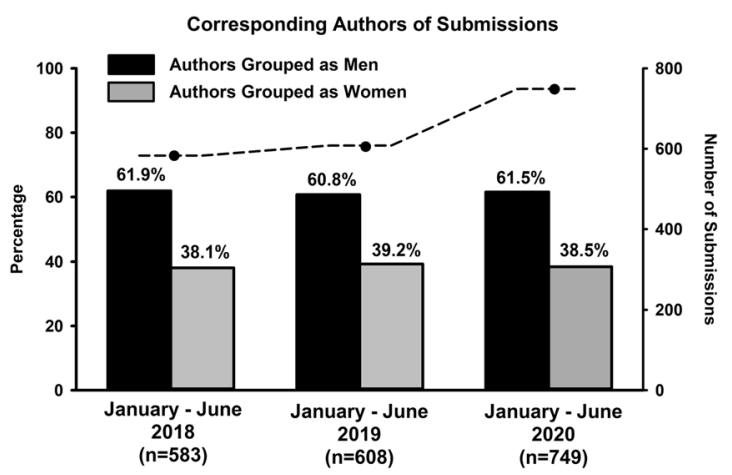

Fig. 1 Corresponding authors of submitted manuscripts during the same 6-month period (January to June) in 2018 and 2019 (before the COVID-19 pandemic) and 2020 (overlapping with the early stages of the COVID-19 pandemic). The percentages of authors identified as men (black bars) or women (gray bars) submitting to NPP as corresponding authors are depicted. The dashed line represents the number of manuscripts submitted during January-June of 2018-2020.

world, the gender representation of corresponding authors was unchanged in comparison with the previous 2 years. These numbers continue to align with the estimated base rate of women in the field of biological psychiatry (40\%).

Focusing on corresponding authors enabled us to use the same methodology as with previous reports [2, 3]; however, we acknowledge that other metrics, including the demographics of first-, middle-, or last-authorship roles, may indeed reveal pandemic-related trends. In addition, these metrics may evolve as the pandemic persists and continues to affect work patterns. Regardless, the leadership teams at NPP and ACNP continue efforts intended to promote mindfulness and increased diversity, equity, and inclusion within the journal and our partner organizations, while also remaining sensitive to concerns of over-burdening individuals from groups who are historically underrepresented in our scientific communities.

\section{FUNDING AND DISCLOSURES}

The authors have formal roles at NPP: CJJ is an Editorial Intern and WAC is the Editor-in-Chief.

\section{DISCLAIMER}

The views and opinions expressed in this manuscript are those of the authors only and do not necessarily represent the views, 
official policy or position of the U.S. Department of Health and Human Services or any of its affiliated institutions or agencies.

\section{ACKNOWLEDGEMENTS}

We are grateful to Gretchen Neigh and Keri Martinowich for early discussions and Jennifer Mahar for providing the raw data for these analyses in ways that maintained the confidentiality of the peer review process.

\section{AUTHOR CONTRIBUTIONS}

CJJ performed the analyses, and CJ and WC wrote the article.

\section{ADDITIONAL INFORMATION}

Publisher's note Springer Nature remains neutral with regard to jurisdictional claims in published maps and institutional affiliations.
Chloe J. Jordan (D) and William A. Carlezon Jr. (DD ${ }^{2}$ ${ }^{1}$ National Institute on Drug Abuse, Baltimore, MD 21224, USA and ${ }^{2}$ Department of Psychiatry, McLean Hospital, Harvard Medical School, Belmont, MA 02478, USA Correspondence: William A. Carlezon Jr. (bcarlezon@mclean.harvard.edu)

\section{REFERENCES}

1. Carlezon WA Jr. NPP statement on racism, discrimination, and abuse of power. Neuropsychopharmacology. 2020;45:1589-90.

2. Jordan CJ, Carlezon WA Jr. NPP (Neuropsychopharmacology): gender balance in journal function. Neuropsychopharmacology. 2018;44:4-8.

3. Jordan CJ, Carlezon WA Jr. NPP (Neuropsychopharmacology): update on gender balance in journal function. Neuropsychopharmacology. 2019; 44:2145-8.

4. Viglione G. Are women publishing less during the pandemic? Here's what the data say. Nature. 2020;581:365-6. 